\title{
Non-simple principally polarised abelian varieties
}

\author{
Paweł Borówka 1
}

Received: 7 April 2015 / Accepted: 20 July 2015 / Published online: 8 August 2015

(C) The Author(s) 2015. This article is published with open access at Springerlink.com

\begin{abstract}
The paper investigates the locus of non-simple principally polarised abelian $g$ folds. We show that the irreducible components of this locus are $\operatorname{Is}_{D}^{g}$, defined as the locus of principally polarised $g$-folds having an abelian subvariety with restricted polarisation of type $D=\left(d_{1}, \ldots, d_{k}\right)$, where $k \leq \frac{g}{2}$. The main theorem produces Humbert-like equations for irreducible components of $\operatorname{Is}_{D}^{g}$ for any $g$ and $D$. Moreover, there are theorems which characterise the Jacobians of curves that are étale double covers or double covers branched in two or four points.
\end{abstract}

Keywords Non-simple abelian varieties · Equations in the Siegel space · Humbert locus · Prym-Tyurin varieties

Mathematics Subject Classification 14K12 · 14H40

\section{Introduction}

A common approach to understand the geometry of the moduli space of principally polarised abelian $g$-folds, denoted $\mathcal{A}_{g}$, is to use ideas coming from the geometry of curves. That is possible because of the Torelli theorem, which says that the Jacobian completely characterises the curve. Because of that, many geometric constructions from the theory of curves give rise to interesting constructions in the theory of Jacobians. One remarkable construction is the Prym construction, which gives a subvariety of a Jacobian for any finite cover of curves. More precisely, every cover of curves $f: C \longrightarrow C^{\prime}$ induces a pullback map $f^{*}: J C^{\prime} \longrightarrow J C$. Therefore $J C$ is a non-simple abelian variety, as it contains im $f^{*}$ and the complementary abelian subvariety, called the Prym variety of the cover.

Paweł Borówka

Pawel.Borowka@uj.edu.pl

1 Institute of Mathematics, Jagiellonian University Krakow, ul. prof. Stanisława Łojasiewicza 6, 30-348 Kraków, Poland 
The motivation behind the results of this paper is to understand the locus of non-simple abelian varieties itself. One can ask:

( ) What does the locus of non-simple principally polarised abelian g-folds look like?

For abelian surfaces, a non-simple abelian surface contains an elliptic curve, and Humbert [9] proved that the locus of non-simple principally polarised abelian surfaces is the union of countably many irreducible surfaces called Humbert surfaces, in the moduli space. The Humbert surfaces are indexed by the degree of the polarisation restricted to an elliptic curve.

In Sect. 2, we propose a definition of the generalised Humbert locus, denoted by $\operatorname{Is}_{D}^{g}$, which is the locus of principally polarised abelian $g$-folds having an abelian subvariety with restricted polarisation of type $D=\left(d_{1}, \ldots, d_{k}\right)$, where $k \leq \frac{g}{2}$. The definition was stated by Debarre [6, p. 259], denoted by $\mathscr{A}_{g^{\prime}, g-g^{\prime}}^{\delta}$. Then he proves irreducibility of $\mathrm{Is}_{D}^{g}$, using irreducibility of some moduli space. The main result of Sect. 2 is Proposition 8 that also states that $\operatorname{Is}_{D}^{g}$ is irreducible. Both ideas of proofs are similar, but the proof presented in this paper is explicit.

Using the fact that every non-simple abelian $g$-fold belongs to some $\operatorname{Is}_{D}^{g}$, we get that the only discrete invariants of the locus of non-simple principally polarised abelian varieties are the dimensions of subvarieties and the type of the restricted polarisation on the smaller one. Moreover, all possibilities of $k \leq \frac{g}{2}$ and $D=\left(d_{1}, \ldots, d_{k}\right)$ can occur.

Note that Guerra [8] considered moduli spaces of embeddings of a pair of abelian varieties of types $D, D^{\prime}$ in an abelian variety of type $E$. He proves some general results but could not verify whether the moduli spaces are actually non-empty. Our result implies that if $E$ is a principal polarisation, for the moduli space to be non-empty, the necessary and sufficient condition is that $D, D^{\prime}$ are complementary polarisation types, see Remark 4 . In particular, we answer one of the questions posted in [8]

Recall that the Siegel space $\mathfrak{h}_{g}=\left\{Z=\left[z_{i j}\right] \in M(g, \mathbb{C}): Z={ }^{t} Z, \operatorname{Im} Z>0\right\}$ is the moduli space of principally polarised abelian varieties with symplectic basis, and $\mathcal{A}_{g}=\mathfrak{h}_{g} / \mathrm{Sp}_{2 g}(\mathbb{Z})$. The main result of the paper is contained in Sect. 3 and provides an explicit answer to Question $(\star)$ :

The locus of non-simple principally polarised abelian $g$-folds is the union of countably many components given by images under the canonical projection $\pi: \mathfrak{h}_{g} \longrightarrow \mathcal{A}_{g}$ of sets of period matrices

$$
\begin{aligned}
\left\{Z_{A}=\left[z_{i j}\right] \in \mathfrak{h}_{g}: z_{i j}\right. & =d_{i} z_{(g+1-i) j}, & i & =1, \ldots, k, \quad j=1, \ldots, k \\
z_{i j} & =0, & i & =k+1, \ldots, g-k, \quad j=1, \ldots, k\},
\end{aligned}
$$

where $k \leq \frac{g}{2}$ and $D=\left(d_{1}, \ldots, d_{k}\right)$ is a possible polarisation type.

Moreover, using results from Sect. 3, one can easily find many other sets of equations that give the same outcome.

The next step of the investigation is to understand the locus of Jacobians that are nonsimple abelian varieties. In full generality, due to the Schottky problem, this task is hard. However, in some specific situations, we have a complete answer. When the genus of the curve is 2 , the answer can be easily extracted from the work of Humbert [9]: the Jacobian of a genus 2 curve $C$ is non-simple and contains an elliptic curve $E$ with the restricted polarisation of type $n$ if and only if the curve $C$ is an $n: 1$ cover of $E$.

In genus 3, the answer is well known and completely analogous. Proposition 4 states that the Jacobian of a genus 3 curve $C$ is non-simple and contains an elliptic curve $E$ with the restricted polarisation of type $n$ if and only if the curve $C$ is an $n: 1$ cover of $E$. However, 
if we restrict our attention to hyperelliptic Jacobians that belong to $\mathrm{Is}_{2}^{3}$, then we find a nice characterisation of étale double covers of genus 2 curves: see Proposition 5 and Corollary 1.

Proposition 5 is generalised in Theorem 7 which, roughly speaking, says that if the Jacobian of a curve contains an abelian subvariety of half the dimension and the type of the restricted polarisation is twice the principal polarisation, then there is a double cover of curves that yields the Jacobian and the subvariety.

\section{Preliminaries}

In this section, we review the well-known facts and set up notation. For more details, see [3].

By an abelian variety, we mean a projective complex torus. An abelian variety $A$ is isomorphic to $\mathbb{C}^{g} / \Lambda_{A}$, where $\Lambda_{A}$ is a lattice of maximal rank. For a $g \times 2 g$ matrix $[Z D]$, the lattice spanned by the column vectors will be denoted by $\langle Z D\rangle$. We can always write $\Lambda_{A}$ in the form

$$
\Lambda_{A}=\langle Z D\rangle,
$$

in such a way that $Z$, called a period matrix of $A$, belongs to the Siegel space

$$
\mathfrak{h}_{g}=\left\{Z=\left[z_{i j}\right] \in M(g, \mathbb{C}): Z={ }^{t} Z, \operatorname{Im} Z>0\right\}
$$

and $D=\operatorname{diag}\left(d_{1}, \ldots, d_{g}\right)$ is a diagonal matrix with positive integer values $d_{i}$ such that $d_{i} \mid d_{i+1}, i=1, \ldots, g-1$. Moreover, on such a variety, we always choose a polarisation of type $D$, usually denoted by $H$.

There are many equivalent definitions of a polarisation. Most of the time, by a polarisation $H$ we mean a Hermitian form on $\mathbb{C}^{g}$ given by a matrix $(\operatorname{Im} Z)^{-1}$ or equivalently an isogeny $\phi_{H}: A \longrightarrow \hat{A}$ with analytic representation given by $(\operatorname{Im} Z)^{-1}$. The exponent of $\phi_{H}$ is denoted by $e(H)$ and called the exponent of the polarisation. By $K(H)$ we denote the kernel of $\phi_{H}$. Using a decomposition for $H$, one proves that $K(H)$ is isomorphic to $\left(\mathbb{Z}_{d_{1}} \times \cdots \times \mathbb{Z}_{d_{g}}\right)^{2}$. On $K(H)$ there exists a multiplicative alternating non-degenerate form

$$
e^{H}\left(w_{1}, w_{2}\right)=\exp \left(-2 \pi i(\operatorname{Im} H)\left(v_{1}, v_{2}\right)\right) \in \mathbb{C}^{*},
$$

where $v_{1}, v_{2}$ are any preimages of $w_{1}, w_{2}$ in $\mathbb{C}^{g}$.

We denote by $\mathcal{A}_{g}=\mathfrak{h}_{g} / \mathrm{Sp}_{2 g}(\mathbb{Z})$ the moduli space of principally polarised abelian $g$-folds and by $\mathcal{A}_{D}$ the moduli space of $D$-polarised abelian varieties. Inside $\mathcal{A}_{g}$, we denote the locus of Jacobians by $\mathcal{J}$ and the locus of hyperelliptic Jacobians by $\mathcal{J H}$.

\subsection{Complementary abelian subvarieties}

In Sect. 2, we will try to understand the locus of non-simple abelian varieties. The idea is to improve the statement of uniqueness in Poincaré's complete reducibility theorem. Therefore we need to recall the following definitions and Poincaré's reducibility theorems. For details we refer to [3].

Definition 1 [3, p. 132] Let $\iota: M \longrightarrow A$ be an abelian subvariety of a principally polarised abelian variety $(A, H)$ and $\hat{\imath}$ be the dual map. Then $\iota^{*} H$ is a polarisation on $M$, denoted also by $\left.H\right|_{M}$. Define the exponent of $M$ by $e(M)=e\left(\iota^{*} H\right)$. Moreover, we define the norm endomorphism of $A$ associated to $M$ by

$$
\mathrm{Nm}_{M}=\iota\left(e(M) \phi_{\iota^{*} H}^{-1}\right) \hat{\iota} \phi_{H} .
$$


$\varepsilon_{M}=\frac{1}{e(M)} \mathrm{Nm}_{M} \in \operatorname{End}_{\mathbb{Q}}(A)$ is called the associated symmetric idempotent.

Conversely for any symmetric idempotent $\varepsilon \in \operatorname{End}_{\mathbb{Q}}(A)$, there exists $n \in \mathbb{N}$ such that $n \varepsilon \in \operatorname{End}(A)$, and we can define the abelian subvariety $A^{\varepsilon}=\operatorname{im}(n \varepsilon)$.

The next theorem is the main tool in proving Poincaré's reducibility theorems.

Theorem 1 [3, Thm 5.3.2] The assignments $M \mapsto \varepsilon_{M}$ and $\varepsilon \mapsto A^{\varepsilon}$ are inverse to each other and give a bijection between the sets of abelian subvarieties of $A$ and symmetric idempotents in $\operatorname{End}_{\mathbb{Q}}(A)$

The main advantage of translating the existence of subvarieties into symmetric idempotents is that the latter have an obvious canonical involution $\varepsilon \mapsto 1-\varepsilon$. This leads to the following definition.

Definition 2 [3, p. 125] Let $A$ be a polarised abelian variety. Then the polarisation induces a canonical involution on the set of abelian subvarieties of $A$ :

$$
M \mapsto N=A^{1-\varepsilon_{M}}
$$

We call $N$ the complementary abelian subvariety of $M$ in $A$, and $(M, N)$ a pair of complementary abelian subvarieties.

In this paper, we often consider products of principally polarised abelian varieties, and therefore, we introduce the following notation.

Definition 3 Suppose $k$ and $g$ are integers with $0<k \leq \frac{g}{2}$, and $D=\left(d_{1}, \ldots, d_{k}\right)$ is a polarisation type. The complementary polarisation type, denoted $\tilde{D}$, is the $(g-k)$-tuple $\left(1, \ldots, 1, d_{1}, \ldots, d_{k}\right)$.

If $\left(M, H_{M}\right)$ and $\left(N, H_{N}\right)$ are polarised abelian varieties of types $D$ and $\tilde{D}$, then, even if it is not written explicitly, we will treat the product $M \times N$ as the $(D, \tilde{D})$-polarised variety with the canonical product polarisation. Strictly speaking, $(D, \tilde{D})$ is not a polarisation type, so one needs to permute the coordinates.

The following proposition shows that indeed the complementary abelian subvariety in the principally polarised abelian variety has a complementary polarisation type

Proposition 1 Let $(A, H)$ be a principally polarised abelian variety. The following conditions are equivalent:

1. there exists $M \subset A$ such that $\left.H\right|_{M}$ is of type $D$.

2. there exists $N \subset A$ such that $\left.H\right|_{N}$ is of type $\tilde{D}$.

3. there exists a pair $(M, N)$ of complementary abelian subvarieties in A of types $D$ and $\tilde{D}$, respectively.

4. there exists a polarised isogeny $\rho: M \times N \longrightarrow A$ with

$$
\operatorname{ker} \rho \cap(M \times\{0\})=\operatorname{ker} \rho \cap(\{0\} \times N)=\{0\} .
$$

Proof The equivalence of conditions (1), (2), (3) follows from the definition and [3, Cor 12.1.5].

(3) $\Rightarrow$ (4) is a consequence of [3, Cor 5.3.6]. The condition on the kernel states that $\left.\rho\right|_{M \times\{0\}}$ and $\left.\rho\right|_{\{0\} \times N}$ are inclusions.

(4) $\Rightarrow$ (3) Let us denote the inclusions by $\iota_{M}=\left.\rho\right|_{M \times\{0\}}$ and $\iota_{N}=\left.\rho\right|_{\{0\} \times N}$. Then $\rho(m, n)=\iota_{M}(m)+\iota_{N}(n)$ and so

$$
\varepsilon_{M}+\varepsilon_{N}=\iota_{M} \phi_{\iota_{M}^{*} H}^{-1} \iota_{M} \phi_{H}+\iota_{N} \phi_{\iota_{N}^{*} H}^{-1} \iota_{N} \phi_{H}=\rho \phi_{\rho^{*} H}^{-1} \hat{\rho} \phi_{H}=\phi_{H}^{-1} \phi_{H}=1
$$


Theorems 2 and 3 are Poincaré's reducibility and complete reducibility theorems.

Theorem 2 [3, Thm 5.3.5] Let $(A, H)$ be a polarised abelian variety and $(M, N)$ a pair of complementary abelian subvarieties of $A$. Then the map

$$
\left(\mathrm{Nm}_{M}, \mathrm{Nm}_{N}\right): A \longrightarrow M \times N
$$

is an isogeny.

Theorem 3 [3, Thm 5.3.7] For an abelian variety A, there is an isogeny

$$
A \longrightarrow A_{1}^{n_{1}} \times \cdots \times A_{r}^{n_{r}}
$$

with simple abelian varieties $A_{i}$ not isogenous to each other. Moreover, the abelian varieties $A_{i}$ and integers $n_{i}$ are uniquely determined up to isogenies and permutations.

\subsection{Symplectic forms on finite abelian groups}

Later, we shall be interested in isotropic subspaces of $K(H)$ and their behaviour under the action of the symplectic group. We will use notation from [3, Sections 6.6 and 8.3.1].

Definition 4 A pair $\left(K(D), e^{D}\right)$ is called a finite symplectic $\mathbb{Z}$ module if $K(D)=\left(\mathbb{Z}_{d_{1}} \times\right.$ $\left.\cdots \times \mathbb{Z}_{d_{k}}\right)^{2}$ with a multiplicative alternating form defined on the standard set of generators $f_{i}$ as follows:

$$
e^{D}\left(f_{i}, f_{j}\right)= \begin{cases}e^{\frac{2 \pi i}{d_{i}}} & \text { if } \mathrm{i}+\mathrm{g}=\mathrm{j} \\ e^{\frac{-2 \pi i}{d_{i}}} & \text { if } \mathrm{j}+\mathrm{g}=\mathrm{i} \\ 1 & \text { otherwise }\end{cases}
$$

Remark 1 For any $i=\{1, \ldots, k\}$, when $d_{i}=1$ we can skip the factor $\mathbb{Z}_{d_{i}}$. In particular, $\left(K(D), e^{D}\right)$ is equal to $\left(K(\tilde{D}), e^{\tilde{D}}\right)$ for complementary polarisation types. However, we will use both notations to distinguish abelian subvarieties.

Example 1 [3, Lem 3.1.4] Let $H$ be a polarisation of type $\left(d_{1}, \ldots, d_{k}\right)$, on an abelian variety $A$ and consider a decomposition $V_{1} \oplus V_{2}$ for $H$. Then

$$
K(H)=K(H)_{1} \oplus K(H)_{2} \text {, with } K(H)_{1} \cong K(H)_{2} \cong \mathbb{Z}_{d_{1}} \times \cdots \times \mathbb{Z}_{d_{k}},
$$

Moreover, $K(H)$ has symplectic form $e^{H}$. The choice of an isomorphism $K(H) \cong K(D)$ is sometimes called a canonical level structure or level D-structure on A. Note that a choice of a period matrix induces a choice of a canonical level structure, see [3, Sec 8.3.1].

Definition 5 Let $\left(K(D), e^{D}\right)$ and $\left(K(\tilde{D}), e^{\tilde{D}}\right)$ be symplectic $\mathbb{Z}$-modules. Then a $\mathbb{Z}$-linear map $f: K(D) \longrightarrow K(\tilde{D})$ is called an antisymplectic map if for all $x, y \in K(D)$, we have

$$
e^{D}(x, y)=-e^{\tilde{D}}(f(x), f(y)) .
$$

Proposition 2 Every antisymplectic map is a bijection and the inverse map is also antisymplectic. Moreover, the space of antisymplectic maps is modelled on $\operatorname{Sp}(K(D), \mathbb{Z})$, i.e. for every antisymplectic $f, g: K(D) \longrightarrow K(\tilde{D})$, we have that $g^{-1} \circ f \in \operatorname{Sp}(K(D), \mathbb{Z})$ and for all $s \in \operatorname{Sp}(K(D), \mathbb{Z})$, the maps $f$ os are antisymplectic. By symmetry, it is also modelled on $\operatorname{Sp}(K(\tilde{D}), \mathbb{Z})$. 
Proof By Eq. (1), if $f(x)=0$, then $e^{D}(x, y)=0$ for every $y$, so $x=0$, which means $f$ is injective. Since groups have the same order, $f$ is bijective. The remaining statements follow from:

$$
\begin{aligned}
& e^{D}\left(g^{-1} \circ f(x), g^{-1} \circ f(y)\right)=-e^{D}(f(x), f(y))=e^{D}(x, y) \\
& e^{D}(f \circ s(x), f \circ s(y))=-e^{D}(s(x), s(y))=-e^{D}(x, y)
\end{aligned}
$$

Proposition 3 Let $\left(K(D), e^{D}\right)$ and $\left(K(\tilde{D}), e^{\tilde{D}}\right)$ be symplectic $\mathbb{Z}$-modules. Consider $\left(K(D) \oplus K(\tilde{D}), e^{D}+e^{\tilde{D}}\right)$. Then the set of graphs of antisymplectic maps is the set of maximal isotropic subspaces of $K(D) \oplus K(\tilde{D})$ intersecting $K(D)$ and $K(\tilde{D})$ only in $\{0\}$.

In particular, all maximal isotropic subspaces of $K(D) \oplus K(\tilde{D})$ intersecting $K(D)$ and $K(\tilde{D})$ only in $\{0\}$ are equivalent under the actions of symplectic groups on $K(D)$ and on $K(\tilde{D})$.

Proof It is obvious that the graph of an antisymplectic map is an isotropic subspace with the desired properties. For the converse, let $Z$ be a maximal isotropic subspace. Then the projections $\pi_{K}(D): Z \longrightarrow K(D)$ and $\pi_{K(\tilde{D})}: Z \longrightarrow K(\tilde{D})$ are bijections. Moreover $\pi_{K(\tilde{D})} \circ \pi_{K}(D)^{-1}$ is antisymplectic and $Z$ is the graph of $\pi_{K(\tilde{D})} \circ \pi_{K}(D)^{-1}$.

The second part of the proposition is a direct application of Proposition 2.

\section{Generalised Humbert locus}

\subsection{Background: Humbert surfaces}

We begin the study of moduli of non-simple abelian varieties with the surface case, by recalling the Humbert surfaces of square discriminant.

Theorem 4 Let p be a positive integer. Let $(A, H)$ be a principally polarised abelian surface. The following conditions are equivalent:

1. there exists an elliptic curve $E \subset A$ such that $\left.H\right|_{E}$ is of type $p$;

2. there exists an exact sequence

$$
0 \longrightarrow E \longrightarrow A \longrightarrow F \longrightarrow 0,
$$

and therefore its dual

$$
0 \longrightarrow F \longrightarrow A \longrightarrow E \longrightarrow 0
$$

such that the induced map $E \times F \longrightarrow A$ is an isogeny of degree $p^{2}$;

3. there exists a pair $(E, F)$ of complementary elliptic curves in A of type $p$;

4. $\operatorname{End}(A)$ contains a primitive symmetric endomorphism $f$ with discriminant $p^{2}$;

5. End $(A)$ contains a symmetric endomorphism $f$ with analytic and rational representations given by

$$
\left[\begin{array}{cc}
0 & 0 \\
-1 & p
\end{array}\right],\left[\begin{array}{cccc}
0 & -1 & 0 & 0 \\
0 & p & 0 & 0 \\
0 & 0 & 0 & 0 \\
0 & 0 & -1 & p
\end{array}\right]
$$


6. $(A, H)$ is isomorphic to an abelian surface defined by a period matrix

$$
\left[\begin{array}{cccc}
p t_{2} & t_{2} & 1 & 0 \\
t_{2} & t_{3} & 0 & 1
\end{array}\right]
$$

with elliptic curves defined by period matrices $\left[t_{2} 1\right]$ and $\left[p t_{3}-t_{2} 1\right]$ embedded as $s \mapsto(p s, s)$ and $s \mapsto(0, s)$.

Proof We have already proved the equivalence of (1), (2) and (3) in Proposition 1 in a more general setting. The equivalence of (4), (5) and (6) is a direct application of [5, Section 4]. In particular, when we set $a=-1, b=p, c=d=e=0$, then equivalence (5) and (6) follows from Corollary 4.2 and equivalence (4) and (6) follows from Corollary 4.6 from [5].

Next, we will show that (3) $\Longrightarrow$ (4) and (6) $\Longrightarrow$ (1).

For the first implication, take $f$ to be the norm endomorphism associated with either elliptic curve. By [3, Thm 5.3.4], $f$ is primitive, symmetric and has characteristic polynomial $f^{2}-p f$. So its discriminant equals $p^{2}$.

For the second implication, to simplify notation, we write $t^{\prime}=\operatorname{Im}(t)$ for any $t \in \mathbb{C}$. Then

$$
\begin{aligned}
& \operatorname{det}(\operatorname{Im} Z)=t_{2}^{\prime}\left(p t_{3}^{\prime}-t_{2}^{\prime}\right), \\
& H=(\operatorname{Im} Z)^{-1}=\operatorname{det}(\operatorname{Im} Z)^{-1}\left[\begin{array}{cc}
t_{3}^{\prime} & -t_{2}^{\prime} \\
-t_{2}^{\prime} & p t_{2}^{\prime}
\end{array}\right] .
\end{aligned}
$$

Denote by $E$ the elliptic curve defined by the period matrix [ $\left.t_{2} 1\right]$ embedded by $\iota_{E}: s \mapsto$ $(p s, s)$. Its analytic representation is given by the matrix $\left[\begin{array}{l}p \\ 1\end{array}\right]$. Then $\phi_{l_{E}^{*} H}=\hat{\iota}_{E} \circ \phi_{H} \circ \iota_{E}$ is defined by

$$
\left[\begin{array}{ll}
p & 1
\end{array}\right] \operatorname{det}(\operatorname{Im} Z)^{-1}\left[\begin{array}{cc}
t_{3}^{\prime} & -t_{2}^{\prime} \\
-t_{2}^{\prime} & p t_{2}^{\prime}
\end{array}\right]\left[\begin{array}{l}
p \\
1
\end{array}\right]=\left[\begin{array}{ll}
p & 1
\end{array}\right]\left[\begin{array}{c}
\frac{p t_{3}-t_{2}^{\prime}}{t_{2}^{\prime}\left(p t_{3}-t_{2}^{\prime}\right)} \\
0
\end{array}\right]=p\left[\left(t_{2}^{\prime}\right)^{-1}\right],
$$

so $\left.H\right|_{E}$ is of type $p$.

Condition (6) of Theorem 4 implies that in $\mathcal{A}_{2}$, the locus of all principally polarised abelian surfaces satisfying the above conditions is the image of the surface given by the equation $t_{1}=p t_{2}$ in $\mathfrak{h}_{2}$, and therefore, it is an irreducible surface in $\mathcal{A}_{2}$.

Definition 6 The locus in $\mathcal{A}_{2}$ of all principally polarised abelian surfaces that satisfy the conditions of Theorem 4 is called the Humbert surface of discriminant $p^{2}$.

Humbert showed more in [9]. He found the equations defining the preimage in $\mathfrak{h}_{2}$ of all Humbert surfaces. To be precise, any 5-tuple of integers without common divisor $(a, b, c, d, e)$ with the same discriminant $\Delta=b^{2}-4 a c-4 d e$ gives us the so-called singular relation

$$
a t_{1}+b t_{2}+c t_{3}+d\left(t_{2}^{2}-t_{1} t_{3}\right)+e=0 .
$$

In other words, the period matrix $Z=\left[\begin{array}{ll}t_{1} & t_{2} \\ t_{2} & t_{3}\end{array}\right] \in \mathfrak{h}_{2}$ is a solution to a singular relation with $\Delta=p^{2}$ if and only if the abelian surface $A_{Z}=\mathbb{C}^{2} /\left(Z \mathbb{Z}^{2}+\mathbb{Z}^{2}\right)$ contains an elliptic curve with restricted polarisation of type $p$.

If we recall that $\mathcal{A}_{2}=\mathfrak{h}_{2} / \operatorname{Sp}(4, \mathbb{Z})$, then it means that all matrices which satisfy the singular equation for some $\Delta=p^{2}$ form a symplectic orbit. Then condition 6 of Theorem 4 says that there always exists a normalised period matrix, i.e. such that

$$
a=-1, b=p, c=d=e=0 .
$$




\subsection{Generalised Humbert locus}

We would like to generalise the notion of Humbert surface to higher dimensions. There are a few immediate problems that arise. Firstly, Humbert surfaces are divisors in $\mathcal{A}_{2}$ globally defined by one equation in $\mathfrak{h}_{2}$, whereas in higher dimensions that is not the case. Secondly, all elliptic curves are essentially canonically principally polarised, whereas in higher dimensions polarisations are much richer.

If we consider an abelian subvariety of an abelian variety, then the two obvious discrete invariants are the dimension of the subvariety and the type of the restricted polarisation. So we define

Definition 7 The generalised Humbert locus of type $D=\left(d_{1}, \ldots, d_{k}\right)$ in dimension $g$, denoted by $\operatorname{Is}_{D}^{g}$, is the locus in $\mathcal{A}_{g}$ of principally polarised $g$-folds $X$ such that there exists a $k$-dimensional subvariety $Z$ of $X$ such that the restriction of the polarisation from $X$ to $Z$ is of type $D$. If $d_{1}=d_{k}$, then we say it is of principal type.

Remark 2 The same definition was proposed by Debarre [6, p. 259], denoted by $\mathscr{A}_{g^{\prime}, g-g^{\prime}}^{\delta}$.

Firstly, some obvious remarks and connections with previously known notions:

1. Every non-simple principally polarised abelian variety belongs to a generalised Humbert locus for some $g$ and $D$.

2. The name comes from the fact that for surfaces, it gives back Humbert surfaces of discriminant $D^{2}$. The word Is is an abbreviation of (polarised) isogenous to a product.

3. Note that principal type does not mean $d_{k}=1$. If $d_{k}=1$, then the isogeny from Proposition 1 is actually an isomorphism and we get only the locus of products of principally polarised abelian varieties. If we restrict the generalised Humbert locus of principal type to Jacobians of smooth curves, then we get Jacobians containing Prym-Tyurin varieties.

The first example of generalised Humbert locus arises in dimension three. The following proposition generalises Barth [2, Proposition 1.8] result about curves on $(1,2)$ polarised surfaces.

Proposition 4 A variety $A \in \mathrm{Is}_{n}^{3}$ is either a product of an elliptic curve with an abelian surface or the Jacobian of a smooth genus 3 curve which is an $n: 1$ cover of an elliptic curve branched in 4 points (counted with multiplicities), and all such Jacobians are contained in $\mathrm{Is}_{n}^{3}$. In other words, the only non-simple Jacobians are Jacobians of covers of elliptic curves.

Proof By [3, Cor 11.8.2], every principally polarised abelian threefold is either a product or a Jacobian so we restrict our attention to Jacobians. If $J C \in \mathrm{Is}_{n}^{3}$, then it contains an elliptic curve, say $E$. Taking the Abel-Jacobi map composed with the dual of the inclusion, we get a map $C \longrightarrow E$. As $\left.H\right|_{E}$ is of type $n$, it is an $n: 1$ cover. Using the Hurwitz formula, we get that it has to be branched in 4 points. Conversely, if we have an $n: 1 \operatorname{cover} \pi: C \longrightarrow E$, then we can have the norm map $\mathrm{Nm}_{\pi}: J C \longrightarrow J E=E$, given by $\mathrm{Nm}_{\pi}(P-Q)=\pi(P)-\pi(Q)$. Moreover, $\operatorname{Nm}_{\pi}\left(\pi^{-1}\left(P^{\prime}-Q^{\prime}\right)\right)=n\left(P^{\prime}-Q^{\prime}\right)$ is a multiplication by $n$, so the restricted polarisation is of type $n$.

Before stating Proposition 5, Theorem 7 and Corollary 2, I would like to note that the results are based on well-known ideas of Prym construction (see [3,10]) and the generalised Torelli Theorem (see [13]), but I was not able to find any exact references in the context of non-simple abelian varieties.

The first result is a characterisation of a 3-dimensional family of Jacobians of étale double covers of genus 2 curves. 
Proposition 5 The locus of Jacobians of étale double covers of genus 2 curves is $\operatorname{Is}_{2}^{3} \cap \mathcal{J H}$.

Proof Let $f: C \longrightarrow C^{\prime}$ be an étale double cover. It is defined by a 2-torsion point in $J C^{\prime}$, say $\eta$. Then $\operatorname{ker}\left(f^{*}\right)=\{0, \eta\}$ (see [1, Ex. B. 14]). Therefore $f^{*} J C^{\prime}=J C^{\prime} /\langle\eta\rangle$ is a $(1,2)$ polarised abelian surface, which is an abelian subvariety of $J C$. Hence $J C \in \mathrm{Is}_{2}^{3}$. To finish the implication, let us note that $C^{\prime}$, being of genus 2 , has to be hyperelliptic and any étale double cover of a genus 2 curve is hyperelliptic. This implication can be easily deduced from the proof of part (a) of [10, Thm 7.1] or from [11].

As for the other implication, let $J C \in \mathrm{Is}_{2}^{3} \cap \mathcal{J H}$. Denote by $E$ an elliptic curve in $J C$ with restricted polarisation of type (2). Denote by $\iota_{E}$ the involution of $C$ which defines the double cover and by $i_{E}$ its extension to $J C$. By construction, $\operatorname{im}\left(1-i_{E}\right)=E$, and therefore, $\epsilon_{E}=\frac{1-i_{E}}{2}$. On the other hand, if we denote by $\iota$ the hyperelliptic involution on $C$, then its extension to $J C$ is $(-1)$. This is because for a branch point $Q$, we have $(P-Q)+(\iota(P)-Q)=0$, being the principal divisor of a pullback of a meromorphic function on $\mathbb{P}^{1}$. Now, $\iota \circ \iota_{E}$ is an automorphism on $C$ and its extension is $-i_{E}$. Denoting by $Z=\operatorname{im}\left(1-\left(-i_{E}\right)\right)$ and $\epsilon_{Z}=\frac{1+i_{E}}{2}$, we immediately get that $\epsilon_{Z}+\epsilon_{E}=1$ and so $(E, Z)$ is a pair of complementary abelian subvarieties of $J C$.

Denote by $\iota_{Z}=\iota \iota_{E}$ and let $C^{\prime}=C / \iota_{Z}$ be the quotient curve with the cover $f: C \longrightarrow C^{\prime}$ given by $P \mapsto\left\{P, \iota_{Z}(P)\right\}$. Then $f^{*}\left(\left\{P, \iota_{Z}(P)\right\}\right)=P+i_{E}(P)$, so $Z=\operatorname{im}\left(f^{*}\right)$.

It is obvious that $\operatorname{dim} J C^{\prime}=\operatorname{dim} Z=2$, so $C^{\prime}$ is of genus 2 and by the Hurwitz Formula $f$ has to be an étale double cover.

The following corollary is an immediate consequence of Proposition 5. It has also been proved in [12] using Galois theory.

Corollary 1 Proposition 5 says that a genus 3 curve is an étale double cover of a genus 2 curve if and only if it is both hyperelliptic and a double cover of an elliptic curve branched in 4 points.

Now, we would like to recall the tools which we use in the proof of Theorem 7.

Theorem 5 (Generalised Torelli theorem [13]) Let C be a smooth curve. Then

$$
\operatorname{Aut}(C)=\left\{\begin{array}{l}
\operatorname{Aut}(J C), \quad \text { if } C \text { is hyperelliptic } \\
\operatorname{Aut}(J C) /(-1), \quad \text { if } C \text { is not hyperelliptic }
\end{array} .\right.
$$

Theorem 6 [3, Prop 11.4.3 and Lem 12.3.1] Let $f: C \longrightarrow C^{\prime}$ be a double cover of smooth curves.

If $f$ is étale, then it is defined by a 2-torsion point on $J C^{\prime}$, say $\eta$, and $f^{*}$ factorises through $J C^{\prime} / \eta$ which is embedded in $J C$. In this case, the restricted polarisation from $J C$ to $J C^{\prime} / \eta$ is of type $(1,2, \ldots, 2)$.

If $f$ is not étale, then $f^{*}$ is injective and the restricted polarisation from $J C$ to $J C^{\prime}$ is twice the principal polarisation on $J C^{\prime}$.

Denote by $\mathcal{D}_{b}^{g}$ the locus of curves of genus $g$ which are double covers ramified in $b$ points and by $\mathcal{J D}_{b}^{g}$ the locus of their Jacobians. Let $\mathcal{H D}_{b}^{g}$ be the locus of hyperelliptic curves of genus $g$ which are double covers ramified in $b$ points and $\mathcal{J H}_{b}^{g}$ their Jacobians. The following theorem is the generalisation of Proposition 5.

Theorem 7 Let $\underline{2}=(2, \ldots, 2)$ be a g-tuple of 2's. Then for odd dimension we have:

$$
\mathrm{Is}_{\underline{2}}^{2 g+1} \cap \mathcal{J}=\mathcal{J D}_{0}^{2 g+1} \cup \mathcal{J D}_{4}^{2 g+1}
$$


and restricting to the hyperelliptic curves, we have:

$$
\mathrm{Is}_{\underline{2}}^{2 g+1} \cap \mathcal{J H}=\mathcal{J H}^{2 g+1}=\mathcal{J H}_{0}^{2 g+1} .
$$

For even dimension, we have:

$$
\mathrm{Is}_{\underline{2}}^{2 g} \cap \mathcal{J}=\mathcal{J D}_{2}^{2 g}, \quad \text { and } \quad \mathrm{Is}_{\underline{2}}^{2 g} \cap \mathcal{J H}=\mathcal{H} \mathcal{H} \mathcal{D}_{2}^{2 g}
$$

Proof The idea of the proof is that the inclusion $\supset$ comes from the Prym construction and $\subset$ comes from the generalised Torelli Theorem.

We will prove the odd dimension case in detail. Let $C \in \mathcal{D}_{0}^{2 g+1} \cup \mathcal{D}_{4}^{2 g+1}$. Let $f$ be the quotient map and $C^{\prime}$ be the quotient curve. If $C \in \mathcal{D}_{4}^{2 g+1}$, then $C^{\prime}$ is of genus $g$ and $f^{*}$ is injective, so $J C^{\prime}$ is embedded in $J C$ with the restricted polarisation of type 2 , whereas for $C \in \mathcal{D}_{0}^{2 g+1}$, we have that $f$ is étale defined by the two torsion points, say $\eta$, and $C^{\prime}$ is of genus $g+1$, so $J C^{\prime} / \eta$ is embedded in $J C$ with the restricted polarisation of type $(1,2, \ldots, 2)$. The complementary polarisation type is $\underline{2}$, so in both cases $J C \in \mathrm{Is}_{\underline{2}}^{2 g+1}$.

Now, let $J C \in \mathrm{Is}_{\underline{2}}^{2 g+1}$. Let $M$ be a subvariety of $J C$ with the restricted polarisation of type $\underline{2}$ and let $N$ be the complementary subvariety to $M$. Then $\mathrm{Nm}_{M}^{2}=2 \mathrm{Nm}_{M}$ and therefore $i_{M}=\left(1-\mathrm{Nm}_{M}\right)$ is an involution of $J C$, because

$$
\left(1-\mathrm{Nm}_{M}\right)^{2}=1-2 \mathrm{Nm}_{M}+\mathrm{Nm}_{M}^{2}=1 .
$$

As $\mathrm{Nm}_{N}=2-\mathrm{Nm}_{M}$, we get that $i_{N}:=\left(1-\mathrm{Nm}_{N}\right)=-i_{M}$ is another involution.

By Theorem 5, one of them comes from the involution on $C$. Assume that $i_{M}$ is the extension of the involution $\iota_{M} \in \operatorname{Aut}(C)$. Let $C^{\prime}=C / \iota_{M}$ and let $f: C \longrightarrow C^{\prime}$ be the quotient map. Then for $c \in C$ we have

$$
f^{*} f(c)=f^{*}\left(\left[c, \iota_{M}(c)\right]\right)=c+\iota_{M}(c)=\mathrm{Nm}_{N}(c) .
$$

Thus im $f^{*}=N$ and as $f^{*}: J C^{\prime} \longrightarrow J C$ is finite onto its image, it means that the genus of $C^{\prime}$ equals

$$
g\left(C^{\prime}\right)=\operatorname{dim}\left(J C^{\prime}\right)=\operatorname{dim}(N)=g+1 .
$$

From the Hurwitz formula, we get that $2(2 g+1)-2=2(2(g+1)-2)+b$, so $b=0$ and therefore $f$ is an étale double cover, so $J C \in \mathcal{J D}_{0}^{2 g+1}$. Analogously, if $i_{N}$ is the extension of the involution on $C$, then $f$ is branched in 4 points, so $J C \in \mathcal{J D}_{4}^{2 g+1}$.

If $C$ is a hyperelliptic curve, then both involutions $i_{M}$ and $i_{N}$ come from involutions on $C$, so $\mathrm{Is}_{2}^{2 g+1} \cap \mathcal{J H} \subset \mathcal{H} \mathcal{H} \mathcal{D}_{0}^{2 g+1}$ and $\mathrm{Is}_{2}^{2 g+1} \cap \mathcal{J H} \subset \mathcal{J} \mathcal{H} \mathcal{D}_{4}^{2 g+1}$ which gives the second part of the theorem.

In the even dimensional case, both $M$ and $N$ are of dimension $g$ and with the restricted polarisation of type $\underline{2}$, and the Hurwitz formula gives $b=2$.

In particular if $C$ is not hyperelliptic and $J C \in \mathrm{Is}_{2}^{2 g}$, then for a pair of complementary subvarieties $(M, N)$ of type 2 , exactly one of them is the Jacobian $J C^{\prime}$ of genus $g$ curve in such a way that $C$ is a double cover of $C^{\prime}$ and the other is the Prym variety of the double cover. If $C$ is hyperelliptic, then both subvarieties are Jacobians and Pryms for each other.

The idea of this proof leads to an interesting observation.

Corollary 2 Let $D=(1, \ldots, 2)$ be a g-tuple with a positive number of 1 's and 2 's. Then

1. $\operatorname{Is}_{D}^{2 g} \cap \mathcal{J}=\emptyset$,

2. $\operatorname{Is}_{D}^{2 g+1} \cap \mathcal{J}=\emptyset$. 
Proof If either of those was non-empty, we would find a pair of complementary subvarieties $(M, N)$ and an involution on $C$ which induces one of the involutions $\left(1-\mathrm{Nm}_{M}\right)$ or $\left(1-\mathrm{Nm}_{N}\right)$. Taking the quotient curve $C^{\prime}$, with quotient map $f$, we would find that $M=f^{*}\left(J C^{\prime}\right)$ or $N=f^{*}\left(J C^{\prime}\right)$.

In the first case, both subvarieties are of dimension $g$, so the Hurwitz formula tells us that $f$ has to be a double cover ramified in two points, which means that the restricted polarisation on $f^{*} J C^{\prime}$ is twice a principal polarisation, a contradiction.

In the second case, the Hurwitz formula states that $2(2 g+1)-2=2\left(2 g\left(C^{\prime}\right)-2\right)+b$, where $b$ is the number of ramification points, and gives two possibilities. If $g\left(C^{\prime}\right)=g+1$, then $b=0$ and we have an étale double cover, and from the Prym construction [3, Thm 12.3.3], we get a contradiction. If $g\left(C^{\prime}\right)=g$, then $b=4$ and again, we get a contradiction because the restricted polarisation on $N$ has to be of type 2 [3, Prop 11.4.3].

There is one more result related to $\mathrm{Is}_{2}^{3}$.

Proposition 6 There is a 1 to 1 correspondence between the set of smooth genus 3 hyperelliptic curves (up to translation) on a general abelian surface $A$ and the set of degree 2 polarised isogenies $A \longrightarrow B$, where $B$ is the Jacobian of a smooth genus 2 curve. In particular, there are exactly three hyperelliptic curves in the linear system of a $(1,2)$ polarising line bundle on a (very) general abelian surface.

Proof Let $\left(J C^{\prime}, \Theta\right)$ be the Jacobian of a smooth genus 2 curve. Let $\rho: A \longrightarrow J C^{\prime}$ be a degree 2 isogeny. Then $\rho^{-1}\left(C^{\prime}\right)$ is a genus 3 hyperelliptic curve on $A$, which is an étale double cover of $C^{\prime}$.

Conversely, let $C$ be a hyperelliptic genus 3 curve on $A$. Then $\mathcal{O}(C)$ is a $(1,2)$ polarising line bundle. From the universal property of Jacobians, there exists a surjective map $f: J C \longrightarrow A$. By [4, Prop 4.3], $\hat{f}$ is an embedding of $\hat{A}$ with restricted polarisation of type $(1,2)$. Therefore $J C \in \mathrm{Is}_{2}^{3}$. As $C$ is also hyperelliptic, Proposition 5 tells us that there exists an étale double cover $C \longrightarrow C^{\prime}$. It is defined by a 2-torsion point, say $\eta$, and there is an embedding of $J C^{\prime} /\langle\eta\rangle$ to $J C$ with the restricted polarisation of type (1,2). As $A$ is general, we have $\hat{A}=J C^{\prime} /\langle\eta\rangle$, and by dualising the quotient map, we obtain a degree 2 polarised isogeny $A \longrightarrow J C^{\prime}$.

The last part follows from the fact that there are exactly three nonzero 2-torsion points in the kernel $K(\mathcal{O}(C))$. A (very) general surface means one for which the resulting principally polarised abelian surface is the Jacobian of a smooth curve.

\subsection{Irreducibility}

The aim of this section is to show that $\operatorname{Is}_{D}^{g}$ is irreducible. This will be an indication that the choice of definition is a good one.

In the proof of this fact, we will use condition (4) of Proposition 1, so we define

Definition 8 Let $M, N, A$ be polarised abelian varieties. An allowed isogeny is a polarised isogeny $\rho: M \times N \longrightarrow A$, such that its kernel has intersection $\{0,0\}$ with $M \times\{0\}$ and $\{0\} \times N$.

Definition 9 Let $\left(M, H_{M}\right),\left(N, H_{N}\right)$ be polarised abelian varieties of type $D$ and $\tilde{D}$. A subgroup $K \subset M \times N$ is called an allowed isotropic subgroup if it is a maximal isotropic subgroup of $K\left(H_{M} \otimes H_{N}\right)$, with respect to $e^{H_{M} \otimes H_{N}}$, such that $K \cap K\left(H_{M}\right)=K \cap K\left(H_{N}\right)=$ $\{0\}$. Note that every maximal isotropic subgroup of $K\left(H_{M} \otimes H_{N}\right)$ has order $\left(d_{1} \cdots d_{k}\right)^{2}$.

Let us recall: 
Proposition 7 [3, Cor 6.3.5] For an isogeny $\rho: Y \longrightarrow X$ and $L \in \operatorname{Pic}(Y)$, the following statements are equivalent:

1. $L=\rho^{*}\left(L^{\prime}\right)$ for some $L^{\prime} \in \operatorname{Pic}(X)$.

2. $\operatorname{ker}(\rho)$ is an isotropic subgroup of $K(L)$ with respect to $e^{L}$.

This leads to an obvious corollary.

Corollary 3 Let A be a principally polarised abelian variety. Let $M, N$ be polarised abelian varieties of type $D$ and $\tilde{D}$. Then

1. If $\rho: M \times N \longrightarrow A$ is an allowed isogeny, then $\operatorname{ker}(\rho)$ is an allowed subgroup of $M \times N$.

2. If $K$ is an allowed subgroup of $M \times N$, then $(M \times N) / K$ is a principally polarised abelian variety and the quotient map $\rho: M \times N \longrightarrow(M \times N) / K$ is an allowed isogeny.

Let us state the main result of this section.

Proposition 8 Let $k, g$ be integers such that $0<k \leq \frac{g}{2}$, and $D=\left(d_{1}, \ldots, d_{k}\right)$ be a polarisation type. Then $\operatorname{Is}_{D}^{g}$ is an irreducible subvariety of $\mathcal{A}_{g}$ of dimension $\left(\begin{array}{c}k+1 \\ 2\end{array}\right)+\left(\begin{array}{c}g-k+1 \\ 2\end{array}\right)$ and codimension $k(g-k)$.

Proof Proposition 1 tells us that $A$ belongs to $\operatorname{Is}_{D}^{g}$ if and only if there exists an allowed isogeny to $A$. Therefore, the idea of the proof is to show that there exists one map from $\mathfrak{h}_{k} \times \mathfrak{h}_{g-k}$ which covers all possible allowed isogenies, and so, $\mathrm{Is}_{D}^{g}$ is the image of an irreducible variety.

The sketch of the proof is as follows. Take polarised abelian varieties $\left(M, H_{M}\right)$ and $\left(N, H_{N}\right)$ of types $D$ and $\tilde{D}$, respectively. Take their product with product polarisation $\left(M \times N, H_{M} \otimes H_{N}\right)$. By [3, Lem 3.1.4], we have $K\left(H_{M}\right) \cong K\left(H_{N}\right)$, and $K\left(H_{M} \otimes H_{N}\right)$, of order $\prod d_{i}^{4}$, is a symplectic $\mathbb{Z}$-module with the non-degenerate symplectic form $e^{H_{M} \otimes H_{N}}$. Therefore, there exists an allowed isotropic subgroup $G \subset K\left(H_{M} \otimes H_{N}\right)$, and by Proposition 3, all such are equivalent under the action of the symplectic group. Hence there exists an allowed isogeny $\rho: M \times N \longrightarrow(M \times N) / G$ and so $\operatorname{Is}_{D}^{g}$ is non-empty. Moreover, the action of the symplectic group on $K\left(H_{M} \otimes H_{N}\right)$ is induced by the symplectic action on $\mathfrak{h}_{g}$, which gives us irreducibility.

To make this more precise, we need to recall that a period matrix of an abelian variety is a choice of symplectic basis of a lattice in its universal cover.

Let $l \leq k$ be the number of integers bigger than 1 in $D$. By the elementary divisor theorem, let $B^{M}=\left\{\lambda_{1}^{M}, \ldots, \lambda_{l}^{M}, \mu_{1}^{M}, \ldots, \mu_{l}^{M}\right\}$ and $B^{N}=\left\{\lambda_{1}^{N}, \ldots, \lambda_{l}^{N}, \mu_{1}^{N}, \ldots, \mu_{l}^{N}\right\}$ be symplectic bases of $K\left(H_{M}\right)$ and $K\left(H_{N}\right)$. Then $B=\left\{B^{M}, B^{N}\right\}$ is a symplectic basis of $K\left(H_{M} \otimes H_{N}\right)$. Let $K_{B}$ be given by the image (that is, the group generated by the columns) of the matrix

$$
K=\left[\begin{array}{cc}
\mathrm{id}_{l} & 0 \\
0 & \mathrm{id}_{l} \\
\mathrm{id}_{l} & 0 \\
0 & -\mathrm{id}_{l}
\end{array}\right]
$$

Then $K_{B}$ is an allowed isotropic subgroup. Moreover, if we change bases using the symplectic action, then $\operatorname{im}(K)$ will always define an allowed isotropic subgroup, and by Proposition 3 , every allowed isotropic subgroup arises in this way.

When we take the universal cover $V$ of $M \times N$, in order to write the period matrix we need to choose a real symplectic basis of $V$. The obvious choice is to enlarge the symplectic basis $B$ to a symplectic basis $\bar{B}$. We need to enlarge the matrix $K$ by zero blocks to a matrix $\bar{K}$, such that its image is equal to $K_{B}$. 
From this discussion, we have found

$$
\Lambda=\left\langle\begin{array}{cccc}
Z(M) & 0 & \operatorname{diag}(D) & 0 \\
0 & Z(N) & 0 & \operatorname{diag}(\tilde{D})
\end{array}\right\rangle, M \times N=\mathbb{C}^{g} / \Lambda,
$$

and a matrix $\bar{K}$, such that $\operatorname{im}(\bar{K})$ is an allowed isotropic subgroup of $M \times N$.

The data defining $\bar{K}$ are discrete, so $\operatorname{im}(\bar{K})$ will be allowed isotropic for any matrices $Z_{k} \in \mathfrak{h}_{k}, Z_{g-k} \in \mathfrak{h}_{g-k}$. Moreover, the symplectic action on $\mathfrak{h}_{k} \times \mathfrak{h}_{g-k}$ gives all possible period matrices; hence, all possible symplectic bases and therefore all possible allowed isotropic subgroups.

Thus we have proved that there exists a global map

$$
\Psi: \mathfrak{h}_{k} \times \mathfrak{h}_{g-k} \ni Z_{k} \times Z_{g-k} \mapsto\left(A_{Z_{k}} \times A_{Z_{g-k}}\right) / \operatorname{im}(\bar{K}) \in \mathcal{A}_{g}
$$

which covers all possible allowed isogenies, that is, for any allowed isogeny $M \times N \longrightarrow A$, there exist period matrices $Z(M)$ and $Z(N)$ such that $\Psi(Z(M) \times Z(N))=A$.

From the construction, it is obvious that $\operatorname{Is}_{D}^{g}$ is the image of the above map, and as the domain is irreducible, it follows that $\operatorname{Is}_{D}^{g}$ is an irreducible variety.

Remark 3 Proposition 8 is stated as a fact in [6, (9.2)] and proved using irreducibility of some moduli space. Both constructions are similar, but the proof presented in this paper is explicit.

There is a generalisation of Humbert surfaces to the moduli of non-principally polarised abelian surfaces. However, in that case, the generalised Humbert surface is no longer irreducible. For details, see [7].

One can also generalise further Proposition 8 to non-principally polarised abelian varieties. Let $D$ be a polarisation type of an abelian $g$-fold. The idea is to define for any polarisation types $D_{1} \in \mathbb{Z}^{k}, D_{2} \in \mathbb{Z}^{g-k}$ the locus $\operatorname{Is}_{D_{1}, D_{2}}^{g, D}$ of $D$-polarised abelian $g$-folds which have a pair of complementary subvarieties of types $D_{1}$ and $D_{2}$. The obvious question is whether $\operatorname{Is}_{D_{1}, D_{2}}^{g, D}$ is non-empty. Using Proposition 7, one can translate the question into one about the existence of isotropic subgroups analogous to the allowed ones. The proof of Proposition 8 can be easily generalised, but one must have in mind that the number of irreducible components of $\operatorname{Is}_{D_{1}, D_{2}}^{g, D}$ will be equal to the number of orbits of such isotropic subgroups. To sum up, the problem can be solved if one can deal with the combinatorics related to special isotropic subgroups in finite symplectic groups. Certainly, this is possible in many cases, such as $(1, p)$-polarised surfaces (see [7]).

Remark 4 In [8], L. Guerra defines the moduli space of embeddings of abelian varieties of types $D, D^{\prime}$ into abelian variety of type $E$ given by an equivalence class of a matrix $[M]$, denoted by $\mathcal{E}_{\delta}[M]$, where $\delta=\left(D, D^{\prime}, E\right)$. He gives a model of such moduli space, being a quotient of Siegel spaces by an action of some group, provided the moduli space is non-empty. If $E$ is a principal polarisation, then there is a natural birational map between $\mathcal{E}_{\delta}[M]$ and $\operatorname{Is}_{D}^{g}$ sending a pair of embeddings to the codomain of both. The map is obviously surjective, and injectivity comes from the fact that on a general non-simple abelian variety there exist exactly two abelian subvarieties. In particular, Is ${ }_{D}^{g}$ does not depend on a matrix $M$, and therefore, we fully answered one of the questions posted in [8, page 21] in case $E$ being principal.

If $E$ is not principal, then the irreducible components of $\operatorname{Is}_{D, D^{\prime}}^{g, E}$ will correspond to a choice of different classes of matrices $[M]$. 


\section{Equations in the Siegel space}

As in the Humbert surface case, we would like to find equations for a locus in $\mathfrak{h}_{g}$ which maps to Is $_{D}^{g}$ in $\mathcal{A}_{g}$. Ideally, we would like to describe the whole preimage of $\operatorname{Is}_{D}^{g}$ which would involve understanding the action of $\operatorname{Sp}(2 g, \mathbb{Z})$ on $\mathfrak{h}_{g}$ and finding good symplectic invariants.

We start by proving an obvious, yet important lemma.

Lemma $1(A, H)=\left(\mathbb{C}^{g} / \Lambda, H\right)$ is non-simple if and only if there exists a $k$-dimensional complex subspace $V$ with $g>k>0$, such that $\Lambda \cap V$ is symplectic of rank $2 k$.

Proof If $A$ is non-simple, then there exists a subvariety $B$ of dimension, say $k$. Taking a universal cover of $A$, we get a lattice $\Lambda$ and a Hermitian form $H$. The preimage of $B$ is a vector subspace, say $V$, and $\Lambda \cap V$ is of rank $2 k$. The restricted polarisation on $B$ is given by restriction of $H$, so $\Lambda \cap V$ has to be symplectic sublattice.

If $V$ is a complex subspace, then we define $B=(V, \Lambda \cap V)$. By assumption, $\left.H\right|_{V}$ is a polarisation, so $B$ is an abelian subvariety and so $A$ is non-simple.

\subsection{Equations of non-simple abelian varieties}

The idea of constructing equations is to choose a sublattice that is symplectic of type $D=$ $\left(d_{1}, \ldots, d_{k}\right)$ and, by applying equations, force it to lie in a complex subspace. To fix notation, let $e_{i}$ denote the basis of $\mathbb{C}^{g}$ and $f_{i}=Z_{A}(i)$ the column vectors of some period matrix $Z_{A}$. Then ${ }^{t} e_{i} \operatorname{Im}\left(Z_{A}\right)^{-1} f_{j}=\delta_{i, j}$ gives a standard matrix of the symplectic form. Choose $g_{i}=d_{i} e_{i}+e_{g-k+i}, i=1, \ldots, k$. Obviously $g_{i}$ 's are primitive, linearly independent and well defined since $k \leq \frac{g}{2}$. Moreover, $\left\{g_{i}, f_{i}, i=1, \ldots, k\right\}$ generates a symplectic sublattice of type $D$. The following equations will force $f_{i}$ 's to lie in the complex subspace generated by $g_{i}$ 's and by Lemma 1 will give the desired outcome. From now on, we start to abuse notation by writing 0 for the block matrix of the correct dimension.

Theorem 8 Let $0<k \leq \frac{g}{2}$ and let $D=\left(d_{1}, \ldots, d_{k}\right)$ be a type of polarisation.

Let $Z_{A}=\left[z_{i j}\right] \in \mathfrak{h}_{g}$ satisfy

$$
\begin{aligned}
& z_{i j}=d_{i} z_{(g-k+i) j}, \quad i=1, \ldots, k, \quad j=1, \ldots, k \\
& z_{i j}=0, \quad i=k+1, \ldots, g-k, \quad j=1, \ldots, k
\end{aligned}
$$

Let $\Lambda_{A}=\left\langle Z_{A} \operatorname{id}_{g}\right\rangle$ and $A=\mathbb{C}^{g} / \Lambda_{A}$. Then $A \in \mathrm{Is}_{D}^{g}$, i.e. there exists an abelian subvariety given by

$$
Z_{M}=\left[\begin{array}{cccc}
z_{11} & z_{12} & \ldots & z_{1 k} \\
z_{12} & z_{22} & \cdots & z_{2 k} \\
\vdots & \vdots & \ddots & \vdots \\
z_{1 k} & z_{2 k} & \cdots & z_{k k}
\end{array}\right], \Lambda_{M}=<Z_{M}, D>, M=\mathbb{C}^{k} / \Lambda_{M}
$$

and an embedding

$$
\begin{aligned}
& \iota_{M}: M \longrightarrow A \\
& \left(x_{1}, \ldots, x_{k}\right)+\Lambda_{M} \longmapsto\left(x_{1}, \ldots, x_{k}, 0, \ldots, 0, \frac{x_{1}}{d_{1}}, \ldots, \frac{x_{k}}{d_{k}}\right)+\Lambda_{A}
\end{aligned}
$$

such that the restricted polarisation is of type $D$. 
Proof Obviously, $Z_{M} \in \mathfrak{h}_{k}$ because it is a principal minor of $Z_{A}$. Moreover $\iota_{M}$ is a welldefined embedding because the images of generators of $\Lambda_{M}$ are given by $\iota_{M}\left(d_{i} e_{i}\right)=d_{i} e_{i}+$ $e_{g-k+i}$ and $\iota_{M}\left(Z_{M}(i)\right)=Z_{A}(i)$, and thus, they are primitive vectors in $\Lambda_{A}$.

To shorten the notation, we will write $Z_{M}(i)$ for the $i$-th column vector of the matrix $Z_{M}$ and $z^{\prime}$ for $\operatorname{Im}(z)$. By $\frac{1}{D}$, we will denote the matrix $\operatorname{diag}\left(\frac{1}{d_{1}}, \ldots, \frac{1}{d_{k}}\right)$.

It remains to compute the restricted polarisation using analytic representations written in block matrices. We have

$$
\begin{aligned}
& {\left[\begin{array}{lll}
\operatorname{id}_{k} & 0 & \frac{1}{D}
\end{array}\right]\left(\operatorname{Im} Z_{A}\right)^{-1}\left[\begin{array}{c}
\operatorname{id}_{k} \\
0 \\
\frac{1}{D}
\end{array}\right] \operatorname{Im} Z_{M}} \\
& =\left[\operatorname{id}_{k} 0 \frac{1}{D}\right]\left[Z_{A}^{\prime}(1) Z_{A}^{\prime}(2) \ldots Z_{A}^{\prime}(g)\right]^{-1}\left[Z_{A}^{\prime}(1) Z_{A}^{\prime}(2) \ldots Z_{A}^{\prime}(k)\right] \\
& =\left[\begin{array}{lll}
\operatorname{id}_{k} & 0 & \frac{1}{D}
\end{array}\right]\left[\begin{array}{c}
\operatorname{id}_{k} \\
0 \\
0
\end{array}\right]=\mathrm{id}_{k},
\end{aligned}
$$

so the restricted polarisation is of type $D$.

Remark 5 Let $\tilde{D}=\operatorname{diag}\left(1, \ldots, 1, d_{1}, \ldots, d_{k}\right)$ be a $(g-k)$-tuple and $\tilde{Z}_{M}=\left[\begin{array}{cc}0 & 0 \\ 0 & Z_{M}\end{array}\right]$ be the zero extension of $Z_{M}$ to a $(g-k) \times(g-k)$ symmetric matrix. Let

$$
X=\left[\begin{array}{cccc}
z_{k+1 k+1} & z_{k+1 k+2} & \ldots & z_{k+1 g} \\
z_{k+1 k+2} & z_{k+2 k+2} & \ldots & z_{k+2 g} \\
\vdots & \vdots & \ddots & \vdots \\
z_{k+1 g} & z_{k+2 g} & \ldots & z_{g g}
\end{array}\right] .
$$

Then

$$
Z_{N}=\tilde{D} X \tilde{D}-\tilde{Z}_{M}
$$

is a symmetric matrix and the complementary abelian subvariety is given by

$$
\Lambda_{N}=<Z_{N}, \tilde{D}>, \quad N=\mathbb{C}^{g-k} / \Lambda_{N}
$$

and

$$
\begin{aligned}
& \iota_{N}: N \longrightarrow A \\
& \left(y_{1}, \ldots, y_{g-k}\right)+\Lambda_{N} \longmapsto\left(0, \ldots, 0, \frac{y_{1}}{\tilde{d}_{1}}, \ldots, \frac{y_{g-k}}{\tilde{d}_{g-k}}\right)+\Lambda_{A}
\end{aligned}
$$

is a well-defined embedding.

To shorten the computations, we will describe only important steps in block matrices. Then

$$
\iota_{N}(\tilde{D})=\left[\begin{array}{c}
0 \\
\operatorname{id}_{g-k}
\end{array}\right] \quad \text { and } \quad \iota_{N}\left(Z_{N}\right)=\left[\begin{array}{c}
0 \\
X \tilde{D}-\tilde{D}^{-1} \tilde{Z}_{M}
\end{array}\right]
$$

is the $g \times(g-k)$ block matrix of the last $(g-k)$ columns of $Z_{A}$ with the last $k$ columns of $Z_{A}$ multiplied by $D$ from the right and having subtracted the first $k$ columns. Therefore, images of generators of $\Lambda_{N}$ are primitive so $\iota_{N}$ is an embedding. Checking that the restricted polarisation is of type $\tilde{D}$ is completely analogous. 
In particular, it is easy to see that the matrix

$$
\left[\begin{array}{ccc}
D & 0 & \mathrm{id}_{k} \\
0 & 2 & \mathrm{id}_{g-k} \\
\operatorname{id}_{k} &
\end{array}\right]
$$

is an explicit example of a matrix that satisfies equations and belongs to $\mathfrak{h}_{g}$, so the set defined by equations is non-empty.

Using Propositions 1 and 8, we can summarise the discussion into the following theorem.

Theorem 9 Let $(A, H)$ be a principally polarised abelian variety and suppose $D$ and $\tilde{D}$ are possible polarisation types of complementary abelian subvarieties. The following conditions are equivalent:

1. there exists an abelian subvariety $M \subset A$ such that $\left.H\right|_{M}$ is of type $D$, i.e. $A \in \mathrm{Is}_{D}^{g}$;

2. there exists a pair $(M, N)$ of complementary abelian subvarieties in $A$ of types $D$ and $\tilde{D}$;

3. $(A, H)$ is isomorphic to an abelian variety defined by the lattice $\Lambda_{A}=\left\langle Z_{A} \mathrm{id}_{g}\right\rangle$, with $Z_{A}=\left[z_{i j}\right] \in \mathfrak{h}_{g}$ satisfying the linear equations

$$
\begin{cases}z_{i j}=d_{i} z_{(g-k+i) j}, & i=1, \ldots, k, \\ z_{i j}=0, & i=k+1, \ldots, g-k, j=1, \ldots, k\end{cases}
$$

Proof (1) $\Leftrightarrow(2)$ is the content of Proposition 1, so we only need to prove that (1) $\Leftrightarrow$ (3). Theorem 8 tells us that the set of abelian varieties with a period matrix satisfying (3) is a subset of $\operatorname{Is}_{D}^{g}$. Moreover, both of them are closed irreducible subvarieties of $\mathcal{A}_{g}$ of codimension $k(g-k)$, which means that they are equal. In other words, the locus

$$
\begin{aligned}
\left\{Z=\left[z_{i j}\right] \in \mathfrak{h}_{g}: z_{i j}\right. & =d_{i} z_{(g+1-i) j}, & i & =1, \ldots, k, j=1, \ldots, k \\
z_{i j} & =0, & i & =k+1, \ldots, g-k, j=1, \ldots, k\}
\end{aligned}
$$

is one of the irreducible components of the preimage of $\operatorname{Is}_{D}^{g}$ in $\mathfrak{h}_{g}$.

Now, we have an explicit answer to the question stated in the introduction.

Corollary 4 The locus of non-simple principally polarised abelian g-folds is a countable union of $\mathrm{Is}_{D}^{g}$, and all $\mathrm{Is}_{D}^{g}$ are images of irreducible loci in $\mathfrak{h}_{g}$ defined by

$$
\begin{aligned}
\left\{Z=\left[z_{i j}\right] \in \mathfrak{h}_{g}: z_{i j}\right. & =d_{i} z_{(g+1-i) j}, & & i=1, \ldots, k, j=1, \ldots, k \\
z_{i j} & =0, & i & =k+1, \ldots, g-k, j=1, \ldots, k\}
\end{aligned}
$$

In Theorem 4, we chose a very simple form of a period matrix with $\Delta=p^{2}$ and generalise it to higher dimensions in Theorem 9. However, it is not hard to make it look more complicated. In particular, the relations between coefficients of a period matrix need not be linear. Proposition 9 will give another set of equations of a preimage of $\operatorname{Is}_{p}^{g}$.

Again let $e_{i}$ 's be a standard complex basis and $f_{i}=Z_{A}(i)$ be column vectors of the Siegel matrix. Take a symplectic sublattice generated by $f_{1}$ and $g_{1}=f_{j}-p e_{1}$ for some $p$ positive integer and $j \neq 1$. Then the assumption that $f_{1}, g_{1}$ are contained in a complex line gives the following proposition. 
Proposition 9 Fix $j \in\{2, \ldots, g\}$ and let $Z_{A}=\left[z_{i k}\right] \in \mathfrak{h}_{g}$ satisfy

$$
z_{11} z_{j i}=z_{1 i}\left(z_{1 j}-p\right), \quad i=2, \ldots g .
$$

Let $\Lambda_{A}=\left\langle Z_{A} \operatorname{id}_{g}\right\rangle$ and $A=\mathbb{C}^{g} / \Lambda_{A}$. Then $A \in \operatorname{Is}_{p}^{g}$, i.e. there exists an elliptic curve

$$
\Lambda_{E}=\left\langle\frac{z_{1 j}-p}{z_{11}} 1\right\rangle, E=\mathbb{C} / \Lambda_{E},
$$

and an embedding

$$
\begin{aligned}
& \iota_{E}: E \longrightarrow A \\
& s+\Lambda_{E} \longmapsto\left(z_{11} s, z_{12} s, \ldots, z_{1 g} s\right)+\Lambda_{A}
\end{aligned}
$$

such that the restricted polarisation is of type $p$.

Proof Without loss of generality, we assume that $\operatorname{Im}\left(\frac{z_{1 j}-p}{z_{11}}\right)>0$. It cannot equal 0 because that would mean that the first and $j$-th rows of $Z_{A}$ are linearly dependent over $\mathbb{R}$.

Obviously $\iota_{E}$ is a well-defined embedding because $\iota_{E}(1)=\left(z_{11}, z_{12}, \ldots, z_{1 g}\right)$ and $\iota_{E}\left(\frac{z_{1 j}-p}{z_{11}}\right)=\left(z_{j 1}-p, z_{j 2}, \ldots, z_{j g}\right)=Z_{A}(j)-p e_{1}$ are primitive vectors in $\Lambda_{A}$.

It remains to compute the restricted polarisation using analytic representations. We need to compute the following real number:

$$
y=\left[\begin{array}{llll}
\bar{z}_{11} & \bar{z}_{12} & \ldots & \bar{z}_{1 g}
\end{array}\right]\left(\operatorname{Im} Z_{A}\right)^{-1}\left[\begin{array}{c}
z_{11} \\
z_{12} \\
\vdots \\
z_{1 g}
\end{array}\right]\left[\left(\frac{z_{1 j}-p}{z_{11}}\right)^{\prime}\right]
$$

As the product of the first three matrices is always real, we will consider $\frac{z_{1 j}-p}{z_{11}}$ instead of its imaginary part and compute $y$ as the imaginary part of the resulting complex number. We have:

$$
{ }^{t} \bar{Z}_{A}(1)\left(\operatorname{Im} Z_{A}\right)^{-1}\left[\begin{array}{c}
z_{11} \\
z_{12} \\
\vdots \\
z_{1 g}
\end{array}\right]\left[\frac{z_{1 j}-p}{z_{11}}\right]={ }^{t} \bar{Z}_{A}(1)\left[Z_{A}^{\prime}(1) \cdots Z_{A}^{\prime}(g)\right]^{-1}\left(Z_{A}(j)-\left[\begin{array}{c}
p \\
0 \\
\vdots \\
0
\end{array}\right]\right) .
$$

Since the matrix of Hermitian form consists of real entries, the imaginary part of the outcome comes from multiplying the imaginary part of one matrix with the real part of the other. Moreover, using a property of the inverse matrix, we get immediately that $y$ is equal to:

$y=\operatorname{Re}\left({ }^{t} \bar{Z}_{A}(1)\right)\left[\begin{array}{c}0 \\ \vdots \\ 1_{j} \\ \vdots \\ 0\end{array}\right]-\left[\begin{array}{llll}1 & 0 & \cdots & 0\end{array}\right]\left(\operatorname{Re}\left(Z_{A}(j)\right)-\left[\begin{array}{c}p \\ 0 \\ \vdots \\ 0\end{array}\right]\right)=\left[\operatorname{Re}\left(z_{1 j}\right)-\left(\operatorname{Re}\left(z_{1 j}\right)-p\right)\right]$,

so the restricted polarisation is of type $p$.

We would like to point out some remarks about the construction of equations and its outcome. 
1. If one chooses a symplectic sublattice to be generated by integer combinations of canonical vector basis, like in Theorem 8, then the analytic representation of the inclusion map has integer coefficients, and therefore, it does not depend on an abelian variety. Moreover, the resulting equations on coefficients of a period matrix will be linear.

2. If one chooses a symplectic sublattice to be generated also by integer combinations of columns of a period matrix, like in Proposition 9, then the analytic representation of the inclusion map depends on an abelian variety. Moreover, the resulting equations on coefficients of a period matrix will be quadratic.

3. The construction is invertible in the following sense: for any $M, N$ abelian varieties of types $D$ and $\tilde{D}$, one can construct an abelian $g$-fold $A$ with period matrix defined by

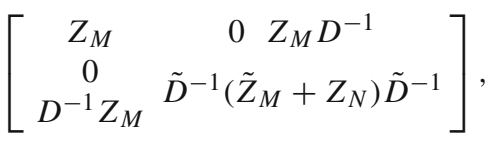

with 0 entries where needed. Then, by Theorem 8, we have $A \in \operatorname{Is}_{D}^{g}$ and it is obvious that $M, N$ are complementary abelian subvarieties in $A$.

4. Note that we only proved irreducibility of 'biggest blocks' in the locus of non-simple principally polarised abelian varieties. If one wants to consider the locus of varieties that contain three different subvarieties with fixed restricted polarisation type, then the irreducibility of such locus is still an unanswered question. It is due to the fact that the restricted polarisation to subvarieties is usually no longer principal, so we cannot apply the above ideas.

In some cases, one can intersect the loci Is ${ }_{D}^{g}$ for different $D$ 's and ask for the irreducibility of the outcome. As an easiest example, consider the locus of principally polarised threefold generated by three elliptic curves with restricted polarisations of types $p, q, r$. If $p, q, r$ are distinct, the locus will be certainly contained and probably almost equal to $\mathrm{Is}_{p}^{3} \cap \mathrm{Is}_{q}^{3} \cap \mathrm{Is}_{r}^{3}$. However, one has to develop new methods to treat the case $p=q=r$.

Acknowledgments Some results of this paper are contained in the $\mathrm{Ph} . \mathrm{D}$. thesis of the author. The author would like to thank his supervisor Gregory Sankaran, University of Bath and Jagiellonian University in Kraków for all support and Olivier Debarre for pointing out results from his paper.

Open Access This article is distributed under the terms of the Creative Commons Attribution 4.0 International License (http://creativecommons.org/licenses/by/4.0/), which permits unrestricted use, distribution, and reproduction in any medium, provided you give appropriate credit to the original author(s) and the source, provide a link to the Creative Commons license, and indicate if changes were made.

\section{References}

1. Arbarello, E., Cornalba, M., Griffiths, P.A., Harris, J.: Geometry of Algebraic Curves, vol. I. Springer, Berlin (1985)

2. Barth, W.: Abelian surfaces with (1,2)-polarization. Adv. Stud. Pure Math. 10, 41-84 (1987)

3. Birkenhake, C., Lange, H.: Complex Abelian Varieties, second edition. Grundlehren der Mathematischen Wissenschaften, 302. Springer, Berlin (2004)

4. Birkenhake, C., Lange, H.: Moduli Spaces of Abelian Surfaces with Isogeny, Geometry and Analysis (Bombay, 1992). Tata Inst. Fund. Res, Bombay, pp. 225-243 (1995)

5. Birkenhake, Ch., Wilhelm, H.: Humbert surfaces and the Kummer plane. Trans. Am. Math. Soc. 355, 1819-1841 (2003)

6. Debarre, O.: Sur les variétés abéliennes dont le diviseur thêta est singulier en codimension 3. Duke Math. J. 56, 221-273 (1988) 
7. van der Geer, G.: Hilbert Modular Surfaces. Ergebnisse der Mathematik und ihrer Grenzgebiete, vol. 3, p. 16. Springer, Berlin (1988)

8. Guerra, L.: Siegel coordinates and moduli spaces for morphisms of Abelian varieties. Annali di Matematica Pura ed Applicata 185, 319-335 (2006)

9. Humbert, G.: Sur les fonctionnes abéliennes singulières, I, II, III. J. Math. Pures Appl. série 5, t. V, pp. 233-350 (1899); t. VI, pp. 279-386 (1900); t. VII, pp. 97-123 (1901)

10. Mumford, D.: Prym Varieties. I, Contributions to Analysis (a Collection of Papers Dedicated to Lipman Bers). Academic Press, New York (1974)

11. Ortega, A.: Variétés de Prym associées aux revêtements $n$-cycliques d'une courbe hyperelliptique. Mathematische Zeitschrift 245, 97-103 (2003)

12. Polizzi, F.: Surfaces of general type with $p_{g}=q=1, K^{2}=8$. Trans. Am. Math. Soc. 358(2), 759-798 (2006)

13. Weil, A.: Zum Beweis des Torellischen Satzes. Nachr. Akad. Wiss. Göttingen. Math. Phys. Kl. IIa. 1957, 33-53 (1957) 